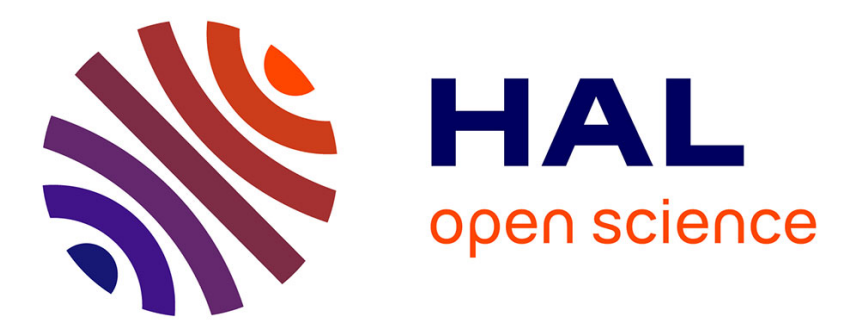

\title{
Impact of Mitral Regurgitation on the Flow in a Model of a Left Ventricle
}

Chloé Papolla, Ahmed Darwish, Lyes Kadem, Régis Rieu

\section{To cite this version:}

Chloé Papolla, Ahmed Darwish, Lyes Kadem, Régis Rieu. Impact of Mitral Regurgitation on the Flow in a Model of a Left Ventricle. Cardiovascular Engineering and Technology, 2020, 11 (6), pp.708-718. 10.1007/s13239-020-00490-y . hal-03219358

\section{HAL Id: hal-03219358 \\ https://hal-amu.archives-ouvertes.fr/hal-03219358}

Submitted on 6 May 2021

HAL is a multi-disciplinary open access archive for the deposit and dissemination of scientific research documents, whether they are published or not. The documents may come from teaching and research institutions in France or abroad, or from public or private research centers.
L'archive ouverte pluridisciplinaire HAL, est destinée au dépôt et à la diffusion de documents scientifiques de niveau recherche, publiés ou non, émanant des établissements d'enseignement et de recherche français ou étrangers, des laboratoires publics ou privés. 


\title{
Impact of Mitral Regurgitation on the Flow in a Model of a Left Ventricle
}

\author{
Chloé Papolla, ${ }^{1,2}$ Ahmed Darwish, ${ }^{3}$ Lyes Kadem, ${ }^{3}$ and Régis Rieu ${ }^{1,4}$ \\ ${ }^{1}$ Aix-Marseille University, Gustave Eiffel University, LBA UMR_T 24, Marseille, France; ${ }^{2}$ Képhalios, part of Affluent Medical \\ Group, Aix-en-Provence, France; ${ }^{3}$ Laboratory of Cardiovascular Fluid Dynamics, Mechanical, Industrial and Aerospace \\ Engineering, Concordia University, Montréal, QC, Canada; and ${ }^{4}$ Laboratoire de Biomécanique Appliquée, Bd. P. Dramard, \\ Faculté de Medecine secteur-Nord, 13916 Marseille Cedex 20, France
}

(Received 1 April 2020; accepted 24 September 2020)

Associate Editor Ajit P. Yoganathan oversaw the review of this article.

\begin{abstract}
Purpose-Mitral regurgitation (MR) is the second most common valve disease in industrialized countries. Despite its high prevalence, little is known about its impact on the flow dynamics in the left ventricle (LV). Because of the interdependence between valvular function and hemodynamics in the heart chambers, an exploration of the dynamics in the LV could lead to a diagnosis of MR. This in vitro study aimed to develop an advanced left heart simulator capable of reproducing several conditions of MR and to evaluate their impact on the LV flow dynamics in terms of flow structures and viscous energy dissipation (VED).

Methods - A simulator, previously developed to test mechanical and biological valves, was upgraded with an original anatomically-shaped mitral valve made from a hydrogel. The valve can be used in healthy or pathological configurations. The nature and severity of the disease was controlled by applying specific strain to the chordae. In this study, in addition to a healthy condition, two different severities of MR were investigated: moderate MR and severe MR. Planar time-resolved particle image velocimetry measurements were performed in order to evaluate the velocity field in the LV and the VED induced by each condition.

Results - Our results showed that MR led to flow disturbances in the LV that were characterized by an increase in mitral inflow velocity and by elevated values of VED. Interestingly VED increased in proportion to the severity of MR and with a dissipation predominating during systole. Conclusion - Considering these results, the introduction of new parameters based on LV VED could provide crucial information regarding the coupling between the mitral valve
\end{abstract}

Address correspondence to Régis Rieu, Laboratoire de Biomécanique Appliquée, Bd. P. Dramard, Faculté de Medecine secteurNord, 13916 Marseille Cedex 20, France. Electronic mail: regis.rieu@univ-amu.fr and the LV and allow for a better stratification of patients with MR.

Keywords-Left ventricle flow, In vitro study, Mitral valve regurgitation, Particle image velocimetry, Viscous energy dissipation.

\begin{tabular}{ll} 
& \multicolumn{1}{c}{ ABBREVIATIONS } \\
MV & Mitral valve \\
LV & Left ventricle \\
MR & Mitral regurgitation \\
PIV & Particle image velocimetry \\
VED & Viscous energy dissipation \\
MRI & Magnetic resonance imaging \\
Echo-PIV & Echocardiographic PIV
\end{tabular}

\section{INTRODUCTION}

The morphology of the native mitral valve (MV) promotes substantial effects in left heart flow dynamics. From a mechanical point of view, it is well known that nature preserves and optimizes the swirling flow in the left ventricle $(\mathrm{LV}) .^{33}$ Left ventricle filling, or diastole, is characterized under healthy conditions by an inflow from the mitral valve generating a vortex structure that was described to optimize energy transport and redirection of the flow towards the aortic valve. $^{18,24,39}$ Left ventricle ejection, or systole, is expected to occur solely through the aortic valve with no flow crossing the mitral valve towards the left atrium..$^{20,21,28}$ The condition where a backflow occurs 
through the mitral valve during systole is referred to as mitral regurgitation (MR). MR is the second most common valvular disease and affects approximately $9 \%$ of the elderly population in industrialized countries. ${ }^{30}$ If the degree of MR is severe, the left atrium tends to enlarge due to the additional stress induced by the increased blood volume exiting the ventricle through the mitral valve. This can be life-threatening and may gradually lead to heart failure. Reliable diagnosis is therefore essential for optimal decision making in terms of mitral valve replacement or repair.

Most of the current literature has focused on the flow dynamics in a healthy left ventricle. A dysfunction of either an element of the mitral apparatus or of the left ventricle may lead to MR and consequently affects intraventricular flow momentum. Any disturbance in diastolic ventricular function is strongly correlated with the development of cardiac disease. ${ }^{24} \mathrm{~A}$ few studies have investigated the flow behaviour under pathological conditions such as ventricular dilatation, ${ }^{4}$ infarction, ${ }^{9}$ or aortic regurgitation. ${ }^{8,37}$ Others have been performed in the context of ventricular repair, particularly in the case of reconstructive valve surgery $^{19}$ or prosthetic mitral valve implantation. ${ }^{6,12,36}$ All these pathologies have an impact on the LV hemodynamics due to its link with the function of the mitral valve.

In the case of MR, the systolic phase is characterized by two outgoing jets, one towards the aortic valve, the other towards the mitral valve. This is expected to influence the flow dynamics in the left ventricle. The evaluation of flow dynamic changes in the ventricle in the presence of MR would be of a great importance to: (1) better understand the detrimental effect of MR and (2) develop robust diagnostic parameters that consider the coupling between the LV and the MR. Many studies have recently introduced the evaluation of viscous energy dissipation as a parameter for assessing ventricular or valvular dysfunction. ${ }^{2,8,34,37}$

Viscous energy dissipation is introduced here as a new parameter that could contribute to an early identification of the flow signature of mitral regurgitation before any irreversible process on the LV occurs. We propose to consider this parameter as a measure of the efficiency of the intraventricular flow to conserve the incoming kinetic energy.

The aim of this study was (1) to demonstrate the ability to reproduce in vitro mitral regurgitation and to explore its hemodynamic consequences on the left ventricle (2) to investigate the effect of moderate and severe mitral regurgitation on the flow dynamics in the left ventricle in terms of flow structures and viscous energy dissipation (VED).

\section{MATERIAL AND METHODS}

\section{Left Heart Simulator}

The duplicator used in this in vitro study mimicked the left human heart. ${ }^{8}$ The LV, the ascending aorta and the left atrium (LA) were replicated using elastic silicone models. The LA 3D model was obtained from a 3D construction of a complete adult human heart using CT and MRI scans (Solid Heart Gen 2 Zygote: American Fork, UT, USA). This model has accurate orientation of the pulmonary veins along with an appendage to ensure realistic flow conditions at the MV inlet. The LV, a simplified and symmetric model, was enclosed in an activation chamber connected to a piston-cylinder assembly. A linear motor (LinMot, NTI AG; Spreitenbach, Switzerland) activates the piston generating a physiological mitral inflow that includes both $\mathrm{E}$ and $\mathrm{A}$ waves. The properties of the circulating fluid have been summarized in Table 1. LV, LA and aortic pressures were measured using a fiber optic pressure sensor (FISO FOP-M260, Canada; range -300 to $300 \mathrm{mmHg}$; resolution $<3 \mathrm{mmHg}$ ). The flow rate was measured using two flowmeters: an ultrasonic flowmeter (T206 Series, Transonic Systems Inc; Ithica, NY) was located downstream of the aortic valve, and a magneto-inductive flowmeter (FMM Series, FMM751002 , ProSense) was located upstream of the pulmonary veins. The ultrasound flowmeter measured the instantaneous LV flow rate through the aorta. The ventricular ejection volume was deduced by integrating the positive part of the flow curve during ventricular systole.

\section{The Mitral Valve}

In order to reproduce the complex geometry and mechanical properties of human MVs, a healthy bileaflet valve made from hydrogel was designed in collaboration with Lifelike BioTissue Inc. (Ontario, Canada) (Fig. 1). This D-shape mitral valve was designed with two leaflets, the anterior is a semi-circular structure and the posterior is divided into three scallops named $\mathrm{P} 1, \mathrm{P} 2$ and $\mathrm{P} 3$.

A total of six chordae were attached to the valve leaflets, two on the anterior, two on scallop P2 and one on scallops P1 and P3. The aorto-mural distance was assessed at $26 \mathrm{~mm}$ and the intercommisural distance at $30 \mathrm{~mm}$. The physiological hemodynamic behavior of this valve used in a left heart simulator is demonstrated here. A short hemodynamic validation of this valve was included in our previous work. ${ }^{32}$ The valve STL file is available as a supplementary material in order to be used by other for experimental or computational fluid dynamics works. In order to control their tension 
TABLE 1. Experimental conditions and fluid properties.

Hemodynamic conditions

Cardiac period: $0.857 \mathrm{~s}$

Heart rate: $70 \mathrm{bpm}$

Mitral flow rate: $4 \pm 0.1 \mathrm{~L} / \mathrm{min}$

Diastolic aortic pressure: $60 \pm 3 \mathrm{mmHg}$

Systolic aortic pressure: $120 \pm 3 \mathrm{mmHg}$
Fluid properties

Water/glycerol ratio 60/40 (by volume) Density $1100 \mathrm{~kg} / \mathrm{m}^{3}$

Dynamic viscosity $4.0 \pm 0.2 \mathrm{cp}$

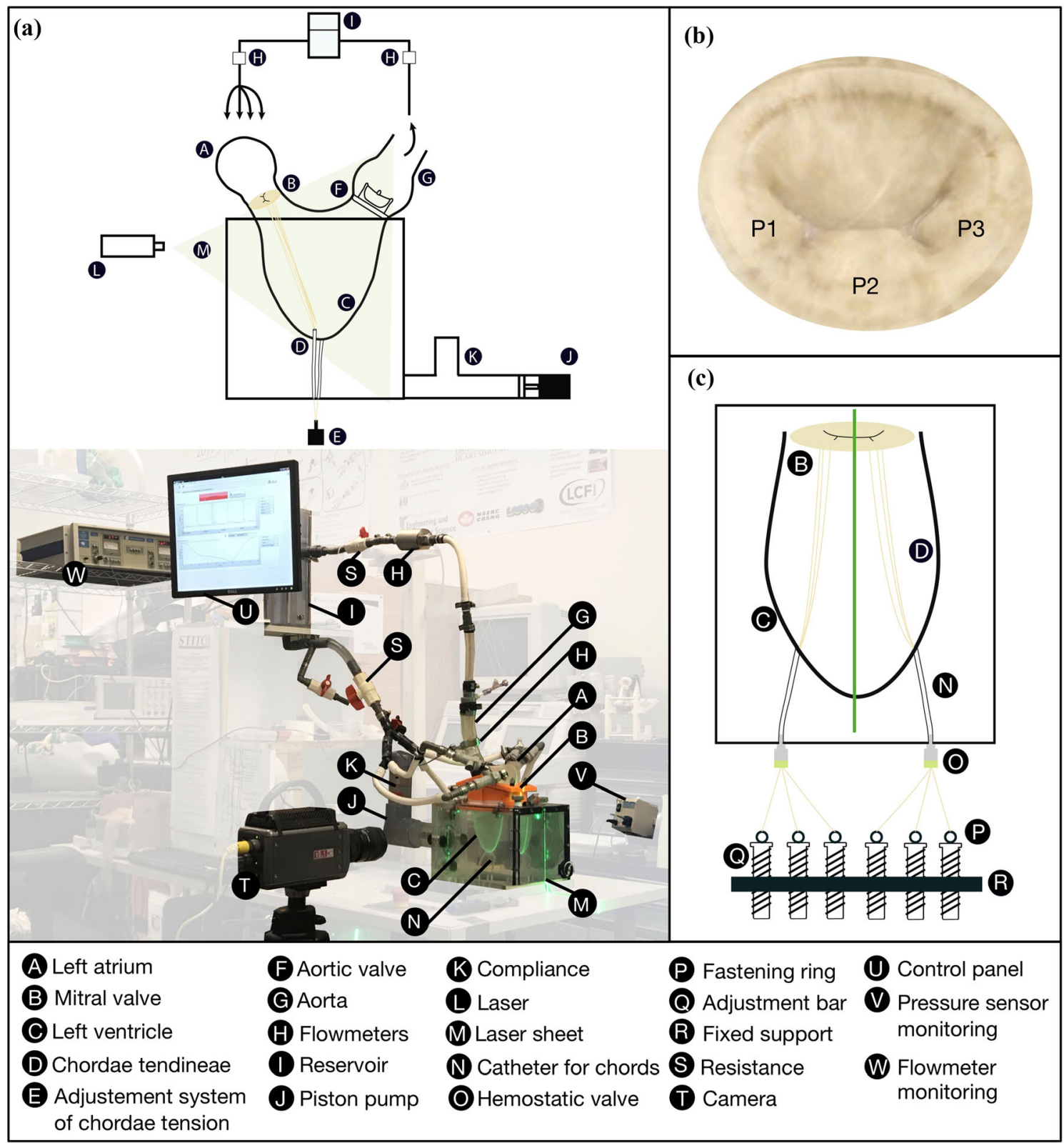

FIGURE 1. (a) Picture and schematic diagram of the left heart simulator. (b) Anatomically-shaped mitral valve. (c) Adjustment system to control the tension of the chordae tendineae. The green line represents the position of the laser sheet. 
while the simulator is in operation, the chordae were directed through catheters to the outside of the activation box down to a control system. The tightness was ensured by two introducers (Radifocus Fr. 6) fixed between the catheters and the ventricular activation box. This control system was inspired by an in vivo setup designed to record the tension applied on the chords during a cardiac cycle reported by Grinberg et al. ${ }^{15}$ In their study, each chord was lengthened by an artificial chordae and connected to a dynamometric bench that recorded individual tension. In our experimental setup, each chord was connected to an adjusting rod allowing the applied tension on each chordae to be increased by tightening. Consequently, pulling on the chords induced a coaptation defect of the mitral valve leaflets and led to an adjustable regurgitation.

\section{Experimental Conditions}

Several scenarios, summarized in Table 2, were investigated in this study. A healthy case was modeled by adjusting the leaflet coaptation optimally preventing any backflow to the atrium. Figure 2 showed the corresponding flow and pressure waveforms as well as the input waveform to the linear motor. Pathological cases were then mimicked by modifying the tension applied on the chordae tendineae. The regurgitant volume was selected as the index of MR severity in order to be consistent with current guidelines. ${ }^{3,29}$ It was inferred as the difference between the aortic stroke volume and the mitral inflow. The mean Reynolds number during mitral inflow was 1111 and the associated Womersley number was 11.9.

The time was normalized as $t^{*}=t / T$, with $t^{*}=0$ representing the beginning of the diastole, $t^{*}=0.6$ the beginning of the systole and $t^{*}=1$ the end of the cycle.

\section{Time-Resolved Particle Image Velocimetry Measurements}

The LV flow field was investigated using a timeresolved particle image velocimetry (TR-PIV) system. The flow seeded with polyamide particles (diameter $50 \mu \mathrm{m}$; density $1030 \mathrm{~kg} / \mathrm{m}^{3}$ ) was illuminated by a $1 \mathrm{~mm}$ width laser sheet formed by a Litron Nd-YLF laser (Litron Laser, England; $10 \mathrm{~mJ}$ output energy at $1 \mathrm{kHz}$; $527 \mathrm{~nm}$ wavelength; repetition rate ranges between 0.2 and $20 \mathrm{kHz}$ ). A high-speed Phantom V9.1 camera (Vision Research, Inc., USA), positioned perpendicularly to the laser sheet, allowed a view of the section intersecting the mitral valve, the aortic valve and the apex of the ventricle. The recorded plane was parallel to the camera and formed by the laser sheet crossing the middle of the apex and the P2 leaflet of the MV. The velocity vectors were computed using the $\mathrm{DaVis}$ 7.2 software (LaVISON GmbH, Germany) by applying a multiple-pass fast Fourier transform cross-correlation to the recorded images. The initial interrogation window was of $64 \times 64$ pixels and the final of $32 \times 32$ pixels with a $50 \%$ overlap. The fourthorder, noise-optimized, hybrid compact-Richardson scheme $^{11}$ was used to calculate the partial derivatives with the discrete velocity data. Each recording was performed three times at a frequency of $1 \mathrm{kHz}$. Various uncertainties are associated with PIV measurements, which are influenced by different sources such as calibration errors, flow characteristics, out-of-plane movements, non-uniform illumination, particle dynamics, light reflection, camera sensitivity, etc. In our experiments, following the recommendations of Ref. 35 . we estimated the uncertainty to be below $5 \%$.

\section{Viscous Energy Dissipation Calculation}

Viscous energy dissipation (VED) represents the dissipation rate of kinetic energy and its irreversible conversion to internal energy by the means of viscous stresses. Typically, higher VED values could be expected when increasing longitudinal or transversal strain rates. Based on recent works of Refs. 8, 33, 37 VED is introduced here as a parameter that could help identifying the signature of a suboptimal coupling between the mitral valve inflow and the left ventricle cavity and thus help to detect early stages of MR before any irreversible process occurs. We propose to consider this parameter as a measure of the efficiency of the intraventricular flow in conserving the incoming kinetic energy. The VED, units of power per unit of depth, was evaluated for each image over all the 2D

TABLE 2. Different mitral valve configurations simulated in this study.

\begin{tabular}{llr}
\hline Simulated cases & Characteristics \\
\hline Healthy & Good leaflet coaptation & Regurgitant volume (mL) \\
Moderate mitral regurgitation & Lack of leaflet coaptation due to a tension on a2-p2 chordae & 50 \\
Severe mitral regurgitation & Lack of leaflet coaptation due to a tension on a2-p2 chordae & 70 \\
\hline
\end{tabular}



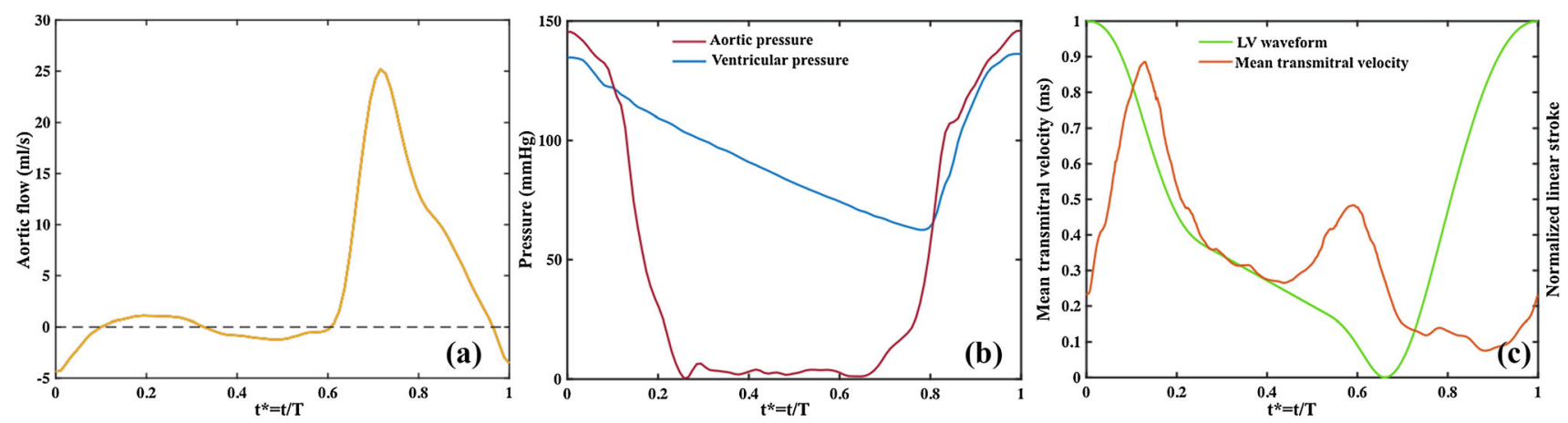

FIGURE 2. (a) Aortic flow waveform. (b) Aortic and ventricular pressure waveforms. (c) Input waveform to the linear motor and transmitral velocity waveform.

view of the ventricular cavity using the following equation:

$$
\mathrm{VED}=\frac{\mu}{2} \int_{A} \sum_{\forall i, j}\left(\frac{\partial u_{i}}{\partial x_{j}}+\frac{\partial u_{j}}{\partial x_{i}}\right)^{2} \mathrm{~d} A
$$

where $\mu$ indicated the dynamic viscosity of the fluid, $u_{i}$ is the velocity in the $x$ direction, $u_{j}$ is the velocity in the $y$ direction, $\mathrm{d} A$ is the interrogation zone area.

\section{RESULTS}

\section{Velocity Fields}

The recorded instantaneous velocity fields at specific key instants during the cardiac cycle corresponding to the diastolic phase are displayed Fig. $3\left(t^{*}=0.10,0.35\right.$, $0.52)$ and Fig. 4 for the systolic phase $\left(t^{*}=0.67,0.82,1\right)$.

During diastole, flow fields are similar in terms of velocity patterns for all cases. Under the healthy condition, the vortex formation time, which is an index of cardiac function defined by Gharib et al., was found to be $T=5.2$, was within the range of $3.3<T<5.5$ obtained for healthy ventricles. ${ }^{14}$ The flow field in all cases is characterized by an incoming jet emerging from the left atrium into the left ventricle. This jet creates then a swirling motion in the LV redirecting the flow towards the left ventricle outflow tract preparing it for ejection through the aortic valve. We noted an increase in the velocity magnitudes through the valve associated with the severity of regurgitation. The Ewave velocity for was $0.9 \mathrm{~m} / \mathrm{s}$ for healthy MR, $1.4 \mathrm{~m} / \mathrm{s}$ for moderate MR while $1.5 \mathrm{~m} / \mathrm{s}$ for the severe case. In the severe MR case, the vortex does not form in the same location as in the other cases. This may be due to the tethering of the mitral valve. A tethered valve causes asymmetric mobility of the leaflets, which will reduce the mobility of the posterior leaflet and this might redirect the flow toward the wall. Further studies are needed to investigate the specific impact of differ- ent types of MR (functional, prolapse, etc.) and the influence of the tethered MV on the skewing of the MV inflow and the vortex formation in the LV.

During systole, significant changes in the flow field with the induced mitral regurgitation were observed. While the flow leaves the left ventricle solely through the aortic valve under the healthy condition, it is split into two flow streams in the presence of mitral regurgitation. This significantly alters the flow in the left ventricle during systole where secondary structures, that were absent under the healthy case, appear in the flow field. During early-systole $\left(t^{*}=0.67\right)$, a longitudinal counter clockwise vortical structure is visible that spans the entire longitudinal length of the LV.

\section{Viscous Energy Dissipation}

The temporal evolution of the space-averaged VED is displayed for all simulated cases on Fig. 5a. In order to isolate the effect of the induced mitral regurgitation on the VED during left ventricle ejection, Fig. 5b represents the average value of the VED over systole only.

For the healthy mitral valve, during the early rapid filling phase, E-wave, after the opening of the mitral valve $\left(t^{*}=0-0.2\right)$, there was a rapid increase in the VED reaching a peak value of $0.17 \mathrm{~J} / \mathrm{m} \mathrm{s}$. This is due to the incoming flow emerging from the mitral valve into the left ventricle. This period was followed by a slow decay in the VED corresponding to diastasis. Finally, during systole a second bump was observed $\left(t^{*}=0.65-0.8\right)$. This temporal evolution in the VED under a healthy condition is consistent with previous in vivo and in vitro findings. ${ }^{1,8}$ To ensure the repeatability of the measurements the VED was calculated for the three recordings of the healthy case. The difference was found non-significant ( $p=0.350)$.

Now, in the presence of MR interesting results were found. MR not only increased significantly $(p<0.05)$ the VED during diastole, but also during systole 


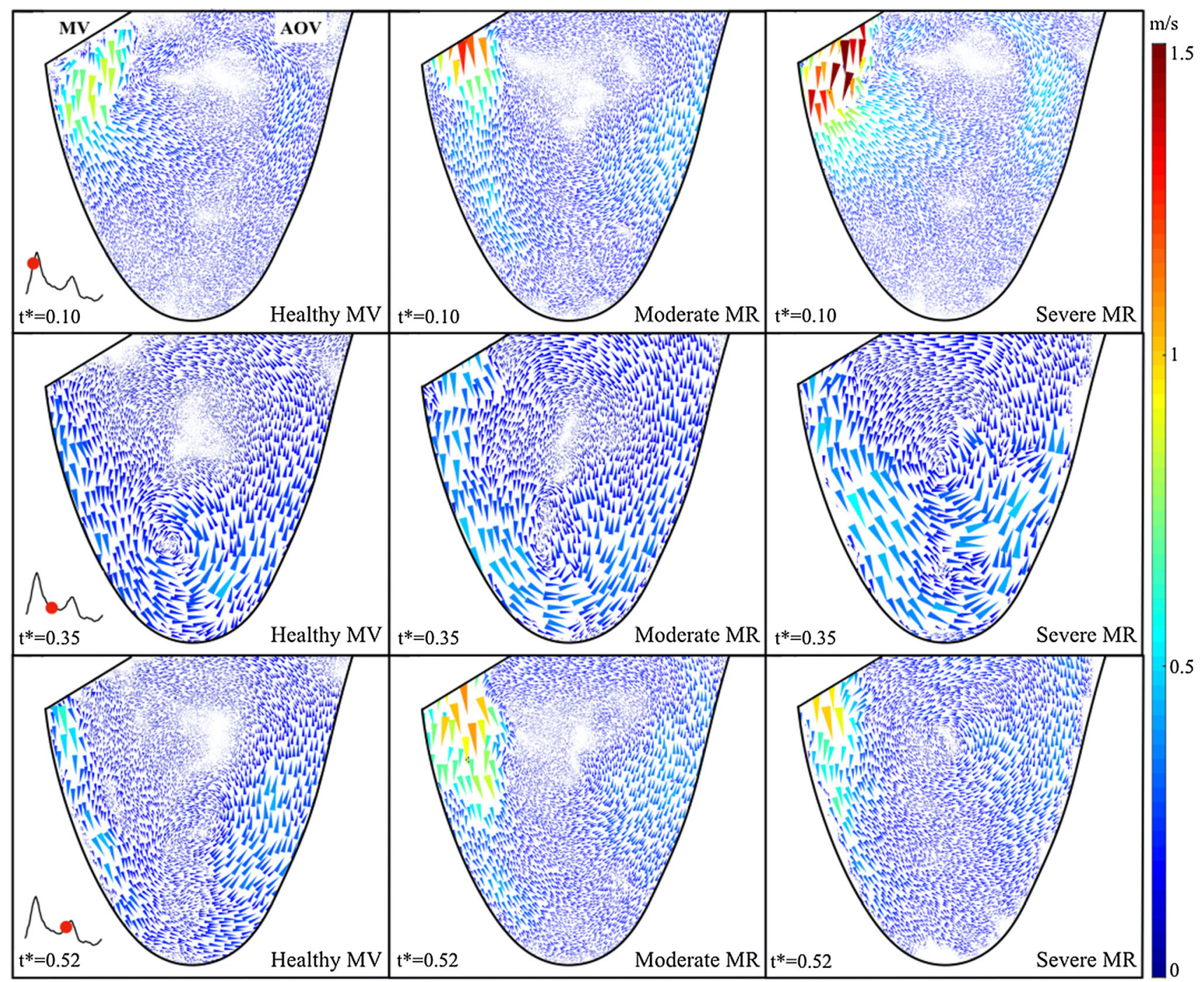

FIGURE 3. Intraventricular flow fields at three selected instants during diastole for the healthy case and for moderate and severe mitral regurgitations.

$(p<0.05)$. In diastole, peak VED was $0.32 \mathrm{~J} / \mathrm{m} \mathrm{s}$ for moderate MR and $0.48 \mathrm{~J} / \mathrm{m}$ s for severe MR. In systole, peak VED was found to follow the same trend, i.e., an increase with MR severity $(0.08 \mathrm{~J} / \mathrm{m} \mathrm{s}$ for the healthy case, $0.34 \mathrm{~J} / \mathrm{m} \mathrm{s}$ for moderate MR and $0.62 \mathrm{~J} /$ $\mathrm{m} \mathrm{s}$ for severe MR). Interestingly enough, peak systolic VED in severe MR was found to exceed the value obtained in diastole. This highlights the presence of a complex, shear intensive, flow that leads to significant energy dissipation during LV ejection.

In terms of average values during systole (Fig. 5b), the VED was found to increase significantly with the severity of MR $(0.05 \pm 0.02 \mathrm{~J} / \mathrm{m}$ s for the healthy case, $0.18 \pm 0.1 \mathrm{~J} / \mathrm{m} \mathrm{s}$ for moderate MR and $0.33 \pm 0.17 \mathrm{~J} /$ $\mathrm{m} \mathrm{s}$ for severe MR; $p<0.01$ for all cases). This increase in the systolic average VED was found to follow a somehow linear trend. But more data are required to confirm this observation.
The effect of temporal resolution on the results was deduced from the calculation of the mean error of the total VED for seven different temporal resolutions ranging from 20 to $1000 \mathrm{~Hz}$. The mean error was found to be a maximum of $4 \pm 2.6 \%$. For the lowest temporal resolutions, considered to be below $150 \mathrm{~Hz}$, the calculated error was less than $1 \%$. The effect of spatial resolution was further evaluated. Halving it (from 1.2 to $2.4 \mathrm{~mm}$ ) induced an error of $78 \%$ of the total VED. These outcomes are similar across all scenarios.

\section{DISCUSSION}

This in vitro research focused on mitral regurgitation by studying its effect on left ventricular function. Moderate and severe mitral regurgitation velocity 
fields were compared to those obtained under healthy conditions while estimating their effect on viscous energy dissipation. The main findings are: (1) the ability of the new design including a realistic mitral valve and chordae to reproduce healthy and different severities of MR has been demonstrated; (2) MR severity increases significantly the VED during both LV filling and ejection phases. For severe MR, the higher values of VED were observed during systole and not during diastole as in the healthy case.

The healthy flow patterns obtained were similar to those observed in in vivo studies on healthy hearts. ${ }^{7}$ Notably, during early-systole, the LV flow pattern has a longitudinal vortical structure. Such structure is noticed when the diastolic flow is directed towards the posterior wall, and the longitudinal vortical structure persist during diastole and early-systolic phase. ${ }^{26}$ The same applies to viscous energy dissipation. ${ }^{16}$ Akiyama et al. explored in vivo ventricular energy performance in healthy adults. They defined reference values of energy loss, kinetic energy and energy performance index to characterize healthy ventricular function and load. $^{2}$

In the event of mitral regurgitation, our results were consistent with the in vivo work of Ref. 38. Indeed, there appears to be a link between the rate of ventricular filling and the severity of mitral regurgitation. Since filling velocities are easily measurable by pulsed Doppler echocardiography, this measurement could be performed and used as a simple screening tool to estimate the progress of mitral regurgitation. However, it is important to take into account that this parameter is valve-centered and does not consider the coupling with the LV cavity. Furthermore, other parameters or pathologies, for example aortic regurgitation, may induce an increase in transvalvular diastolic velocity. ${ }^{31}$ Therefore, this measure alone would not provide a reliable and accurate diagnosis but could be used as part of a multi-parametric assessment.

Soyama Yuko et al. reported on the evolution of intraventricular energy loss in 64 patients with mitral insufficiency. ${ }^{40}$ Vector flow mapping using color Doppler ultrasound allowed the quantification of energy losses in the left ventricle; these were significantly higher in patients with functional mitral insufficiency than in healthy control patients. Our in vitro values of the evolution of the average VED in systole calculated for different MR severities are comparable to their reported in vivo results. Moreover, a parallel can be drawn, with the findings obtained in studies that evaluated the impact of aortic regurgitation on ventricular energy losses. The in vivo investigation conducted by Stugaard et al. using vector flow mapping showed that diastolic VED increases in aortic regurgitation proportional to its severity. ${ }^{37}$ These results were further reinforced with the in vitro PIV study performed by Di Labbio et al. ${ }^{8}$ Both studies demonstrated that an increase in diastolic VED may be associated with aortic regurgitation. Our study, on the other hand, showed that an increase in systolic VED may be an indicator of mitral regurgitation.

Before this work can be translated to clinical practice we sought to evaluate the sensitivity of VED computation to reduced temporal and spatial resolutions. The resolutions of most commonly used clinical modalities are indeed much lower than those obtained in vitro using time-resolved particle image velocimetry. For this purpose, both spatial and temporal resolutions of our recordings were reduced in order to get closer to those typically obtained using echocardiographic PIV (Echo-PIV) (temporal resolution: 50 to $120 \mathrm{~Hz}$; spatial resolution: 2 to $3 \mathrm{~mm}$ ) and PhaseContrast magnetic resonance imaging (MRI) (temporal resolution: 15 to $50 \mathrm{~Hz}$; spatial resolution: 1.5 to $3 \mathrm{~mm}$ ). Our findings showed that a reduction in temporal resolution does not significantly affect the calculations of VED. However, it is significantly influenced by the spatial resolution. It is therefore essential to take into account the spatial resolution of the imaging modality used in order to have a reliable assessment.

\section{Clinical Impact: See Beyond the Mitral Valve}

The severity of mitral regurgitation is evaluated with a multi-parametric evaluation using quantitative, qualitative and semi-quantitative echocardiographic measurements. ${ }^{3,29}$ Regurgitant volume, regurgitation fraction and effective regurgitant orifice area are quantitative parameters determined using quantitative methods, such as the volumetric method or the proximal isovelocity surface area (PISA) method. The PISA method is considered to be the reference for MR quantification and guidelines underlines that it should be performed whenever possible. ${ }^{22,41}$ The accuracy of this Doppler echocardiographic method remains criticized due to its sensitivity to the geometry of the regurgitating orifice. It may underestimate the severity of MR if inappropriate assumptions are chosen. 5,17,23 Although its formula is further improved for specific MR geometries (oblong MR, multiple jets MR), ${ }^{25,42}$ the PISA method is mainly based on a valve evaluation.

Since the thresholds for quantifying secondary MR are still discussed, given that the European guidelines differ from the American guidelines, new parameters could be considered for the quantification of MR., ${ }^{3,10}$ Assessment of the severity of MR should not be limited to a single valve-oriented parameter, and we must essentially look beyond the valve. One way to do this is 


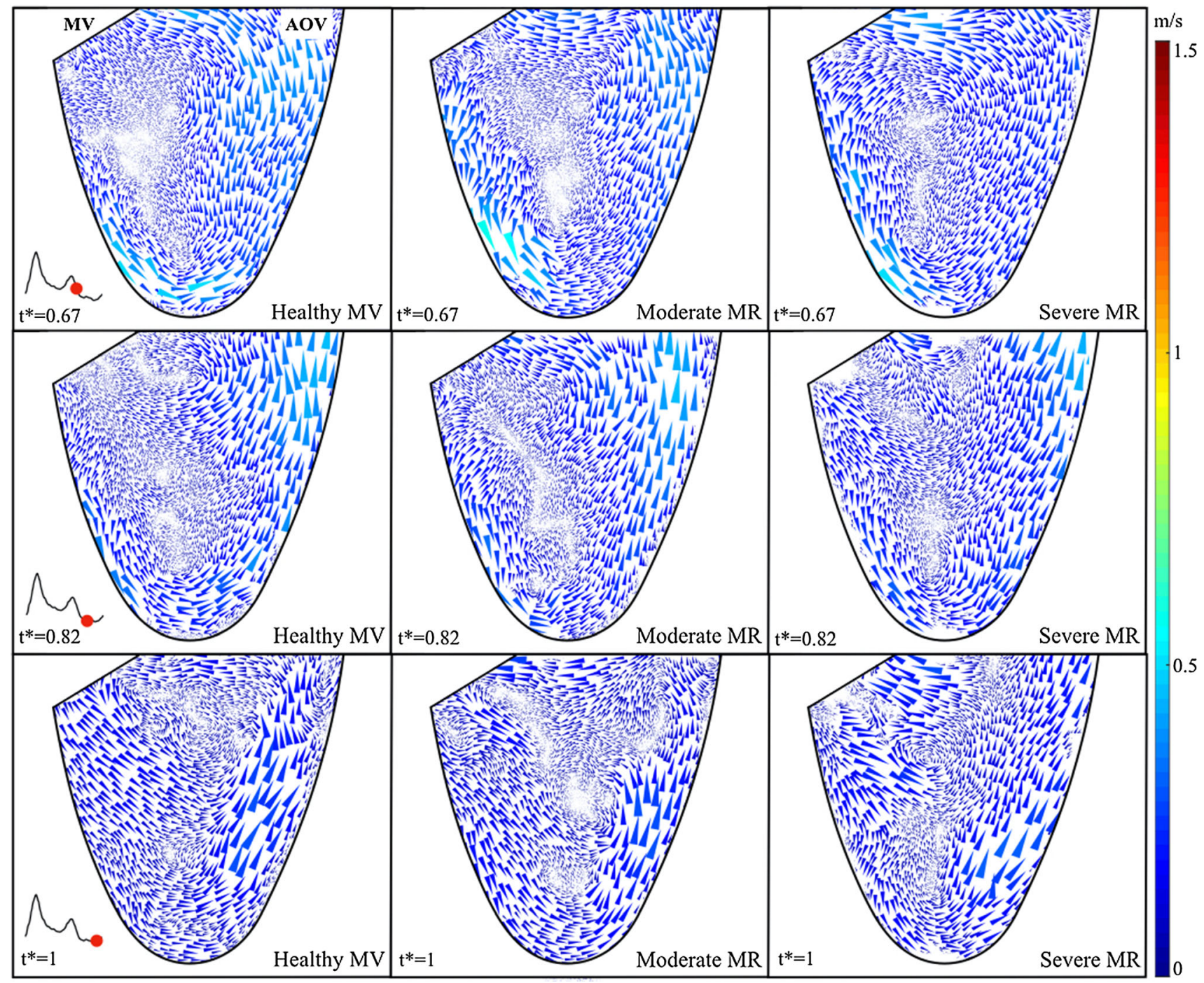

FIGURE 4. Intraventricular flow fields at three selected instants during systole for the healthy case and for moderate and severe mitral regurgitations.

to examine the flow in the left ventricle. Therefore, the VED calculation was introduced to provide essential information regarding the effect of MR on LV performance.

Because of the relationship between LV performance and the development of mitral regurgitation, accessing to velocity fields is clinically relevant. Now, clinicians have access to this information through echocardiography or phase contrast magnetic resonance imaging (PC-MRI). With the growing interest in speckle tracking and echocardiographic particle image and velocimetry (echo-PIV), which allow a non-invasive access to the velocity fields in the heart chambers, ${ }^{13}$ it is timely to develop more robust fluid dynamic-based parameters that provide information beyond the mitral valve function. The VED calculation is a new approach capable of providing important information on the impact of MR on left ventricular performance. In conjunction with the existing valverelated parameters, it shall allow a multi-parametric approach that will significantly improve the evaluation of MR in patients and optimize clinical decisions.

\section{Limitations}

Because in vitro studies require simplifying assumptions and do not account for all physiological conditions, the study was subject to several limitations associated with experimental research using cardiac duplicators. Expansions and contractions of the mitral annulus throughout the cardiac cycle were not modeled. The anatomical relationship between the aortic and mitral valves is such that there is fibrous continuity therebetween. In this experiment, the two valves were separated by a distance, so that this physiologically observed aorto-mitral continuity was not preserved. 

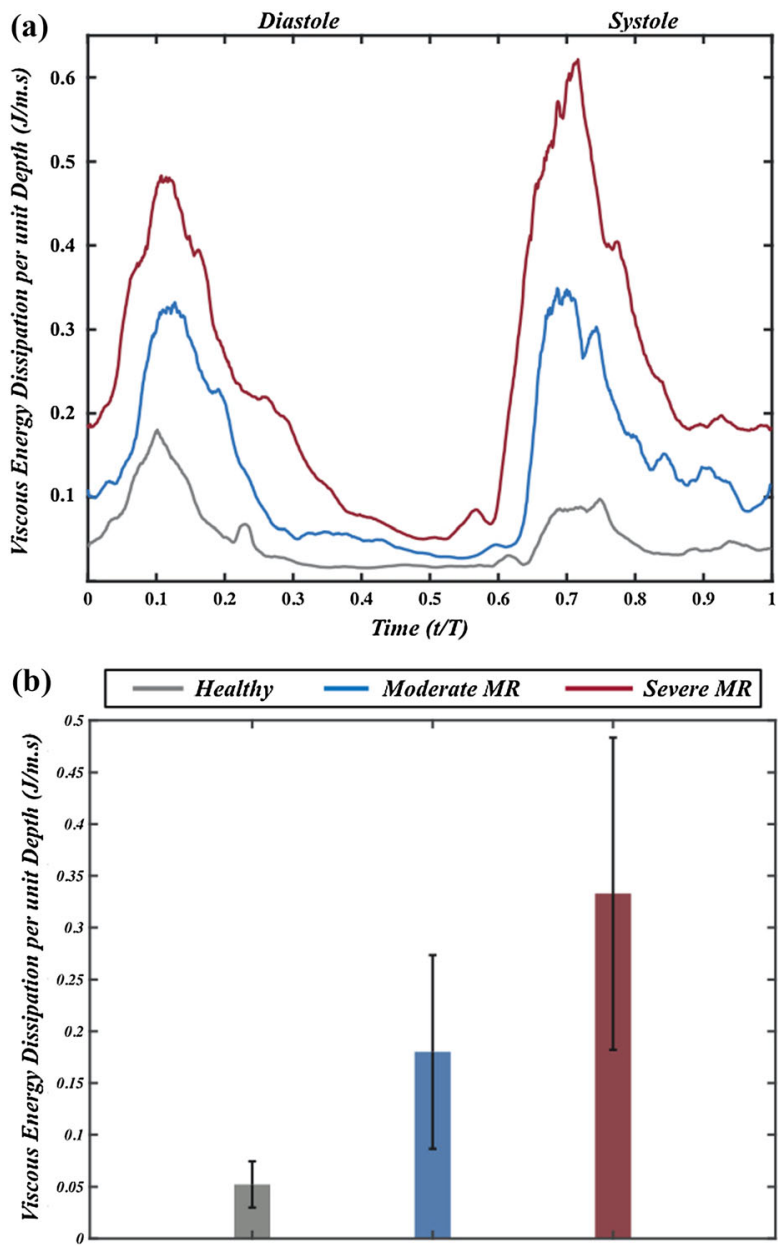

FIGURE 5. (a) Temporal evolution of space-averaged viscous energy dissipation per unit of depth for all the healthy case and for moderate and severe mitral regurgitations. (b) Systolic average for the healthy case and for moderate and severe mitral regurgitations.

It is also well known that functional mitral regurgitation leads to an increase in afterload, which may result in the development of ventricular remodeling in response to increased stress on its wall. Ventricular remodeling refers to a change in the architecture of the ventricle. It includes annular dilatation, spherization of the cavity, off-axis of the papillary muscles, and restriction of leaflet movement in systole. ${ }^{27}$ By using a tethered mitral valve to simulate functional mitral regurgitation, neither these ventricular effects nor changes in atrial pump function (expressed as an increase in the $E / A$ ratio) were considered in this study.

The in vivo study of an isolated pathology is not a trivial matter since it is time-consuming, expensive and involves large patient recruitment. In addition, the resolutions of current imaging techniques still suffer from many limitations and do not allow a reliable and accurate flow study. Although in vitro studies require simplifying assumptions and do not consider all physiological conditions, cardiac simulators are powerful tools. By allowing independent control conditions, they allow analyzing the independent contribution of each factor related to the patient's anatomy, hemodynamic conditions, pathologies, implanted devices and prostheses, etc.

Although the three-dimensional hemodynamics of the left ventricle were simulated in this study, velocity recordings were limited to a $2 \mathrm{D}$ view. With planar PIV, the acquired velocity fields only capture two of the three velocity components. Therefore, it should be noted that the measurements in this study are a twodimensional slice of a three-dimensional flow. However, the chosen plan is conventionally used in clinical practice such in echocardiographic measurements. In addition, due to the symmetrical shape of the LV, the chosen mid-plane is expected to have limited out-ofplane particle motion. Further studies are however still needed to evaluate the complete 4D flow structures by using tomographic PIV.

The objective of this research was to introduce new parameters to study the mitral insufficiency, such as viscous energy dissipation, as potential parameters to be used clinically. Given all these limitations and the limited number of types and severities of mitral regurgitation simulated in this study, these parameters could then be tested in vivo on a larger population, including all realistic cases.

\section{CONCLUSION}

To the best of our knowledge, this is the first study that evaluates in vitro the effect of mitral regurgitation on the left ventricle. First, it introduced a promising simulator capable of mimicking several types and severities of mitral pathologies. Different degrees of regurgitation were modeled thanks to the possibility of adjusting the leaflet coaptation zones. The observation of velocity fields in pathological conditions suggested that regurgitation resulted in an additional vortical structure during flow ejection compared to the movement of healthy flow, which had a major impact on intraventricular hemodynamics. The subsequent assessment of the viscous energy loss was of importance because it correlated the value with the severity of regurgitation. The viscous energy dissipation could be an indicator of mitral regurgitation and on the coupling between the mitral valve and the left ventricle. New thresholds could be defined in a clinical study taking into account the temporal and spatial resolution of the imaging modalities. 


\section{ELECTRONIC SUPPLEMENTARY MATERIAL}

The online version of this article (https://doi.org/10. 1007/s13239-020-00490-y) contains supplementary material, which is available to authorized users.

\section{CONFLICT OF INTEREST}

The authors declare that they have no conflict of interest.

\section{REFERENCES}

${ }^{1}$ Akiyama, K., S. Maeda, T. Matsuyama, A. Kainuma, M. Ishii, Y. Naito, M. Kinoshita, S. Hamaoka, H. Kato, Y. Nakajima, N. Nakamura, K. Itatani, and T. Sawa. Vector flow mapping analysis of left ventricular energetic performance in healthy adult volunteers. BMC Cardiovasc. Disord. 2017. https://doi.org/10.1186/s12872-016-0444-7.

${ }^{2}$ Barker, A. J., P. van Ooij, K. Bandi, J. Garcia, M. Albaghdadi, P. McCarthy, R. O. Bonow, J. Carr, J. Collins, S. C. Malaisrie, and M. Markl. Viscous energy loss in the presence of abnormal aortic flow. Magn. Reson. Med. 72:620-628, 2014. https://doi.org/10.1002/mrm.24962.

${ }^{3}$ Baumgartner, H., V. Falk, J. J. Bax, M. De Bonis, C. Hamm, P. J. Holm, B. Iung, P. Lancellotti, E. Lansac, D. Rodriguez Muñoz, R. Rosenhek, J. Sjögren, P. Tornos Mas, A. Vahanian, T. Walther, O. Wendler, S. Windecker, J. L. Zamorano, and ESC Scientific Document Group. ESC/EACTS guidelines for the management of valvular heart disease. Eur. Heart J. 38(2017):2739-2791, 2017. h ttps://doi.org/10.1093/eurheartj/ehx391.

${ }^{4}$ Bolger, A. F., E. Heiberg, M. Karlsson, L. Wigström, J. Engvall, A. Sigfridsson, T. Ebbers, J.-P. E. Kvitting, C. J. Carlhäll, and B. Wranne. Transit of blood flow through the human left ventricle mapped by cardiovascular magnetic resonance. J. Cardiovasc. Magn. Reson. 9:741-747, 2007. h ttps://doi.org/10.1080/10976640701544530.

${ }^{5}$ Buck, T., C. H. P. Jansen, A. P. Yoganathan, R. A. Levine, and M. D. Handschumacher. Hemisphere versus hemiellipse: when is each most accurate for proximal isovelocity calculation of regurgitant flows. J. Am. Coll. Cardiol. 31:385, 1998. https://doi.org/10.1016/S0735-1097(98)8002 5-9.

${ }^{6}$ Chandran, K. B., R. Schoephoerster, and K. C. Dellsperger. Effect of prosthetic mitral valve geometry and orientation on flow dynamics in a model human left ventricle. J. Biomech. 22:51-65, 1989. https://doi.org/10.1016/ 0021-9290(89)90184-x.

${ }^{7}$ Cimino, S., G. Pedrizzetti, G. Tonti, E. Canali, V. Petronilli, L. De Luca, C. Iacoboni, and L. Agati. In vivo analysis of intraventricular fluid dynamics in healthy hearts. Eur. J. Mech. B 35:40-46, 2012. https://doi.org/10. 1016/j.euromechflu.2012.03.014.

${ }^{8}$ Di Labbio, G., and L. Kadem. Jet collisions and vortex reversal in the human left ventricle. J. Biomech. 78:155160, 2018. https://doi.org/10.1016/j.jbiomech.2018.07.023.

${ }^{9}$ Domenichini, F., and G. Pedrizzetti. Intraventricular vortex flow changes in the infarcted left ventricle: numerical results in an idealised 3D shape. Comput. Methods Biomech. Biomed. Eng. 14:95-101, 2011. https://doi.org/10.10 80/10255842.2010.485987.
${ }^{10}$ Enriquez-Sarano, M., C. W. Akins, and A. Vahanian. Mitral regurgitation. Lancet 373:1382-1394, 2009. https://d oi.org/10.1016/S0140-6736(09)60692-9.

${ }^{11}$ Etebari, A., and P. P. Vlachos. Improvements on the accuracy of derivative estimation from DPIV velocity measurements. Exp. Fluids 39:1040-1050, 2005. https://doi. org/10.1007/s00348-005-0037-1.

${ }^{12}$ Faludi, R., M. Szulik, J. D'hooge, P. Herijgers, F. Rademakers, G. Pedrizzetti, and J.-U. Voigt. Left ventricular flow patterns in healthy subjects and patients with prosthetic mitral valves: an in vivo study using echocardiographic particle image velocimetry. J. Thorac. Cardiovasc. Surg. 139:1501-1510, 2010. https://doi.org/10.1016/j.jtcvs. 2009.07.060.

${ }^{13}$ Garcia, D., P. Lantelme, and E. Saloux. Introduction to Speckle Tracking in Cardiac Ultrasound Imaging. London: Institution of Engineering and Technology, 2018.

${ }^{14}$ Gharib, M., E. Rambod, A. Kheradvar, D. J. Sahn, and J. O. Dabiri. Optimal vortex formation as an index of cardiac health. Proc. Natl. Acad. Sci. USA 103:6305-6308, 2006. h ttps://doi.org/10.1073/pnas.0600520103.

${ }^{15}$ Grinberg, D., P.-J. Cottinet, S. Thivolet, D. Audigier, J.-F. Capsal, M.-Q. Le, and J.-F. Obadia. Measuring chordae tension during transapical neochordae implantation: toward understanding objective consequences of mitral valve repair. J. Thorac. Cardiovasc. Surg. 2018. https://doi.org/ 10.1016/j.jtcvs.2018.10.029.

${ }^{16}$ Hayashi, T., K. Itatani, R. Inuzuka, N. Shimizu, T. Shindo, Y. Hirata, and K. Miyaji. Dissipative energy loss within the left ventricle detected by vector flow mapping in children: normal values and effects of age and heart rate. $J$. Cardiol. 66:403-410, 2015. https://doi.org/10.1016/j.jjcc.20 14.12.012.

${ }^{17}$ Iwakura, K., H. Ito, S. Kawano, A. Okamura, T. Kurotobi, M. Date, K. Inoue, and K. Fujii. Comparison of orifice area by transthoracic three-dimensional doppler echocardiography versus proximal isovelocity surface area (PISA) method for assessment of mitral regurgitation. Am. J. Cardiol. 97:1630-1637, 2006. https://doi.org/10.1016/j.a mjcard.2005.12.065.

${ }^{18}$ Khalafvand, S. S., E. Y.-K. Ng, L. Zhong, and T.-K. Hung. Three-dimensional diastolic blood flow in the left ventricle. J. Biomech. 50:71-76, 2017. https://doi.org/10.1 016/j.jbiomech.2016.11.032.

${ }^{19}$ Khalafvand, S. S., L. Zhong, and E. Y. K. Ng. Threedimensional CFD/MRI modeling reveals that ventricular surgical restoration improves ventricular function by modifying intraventricular blood flow. Int. J. Numer. Methods Biomed. Eng. 30:1044-1056, 2014. https://doi.org/ 10.1002/cnm.2643.

${ }^{20}$ Kilner, P. J., G.-Z. Yang, A. J. Wilkes, R. H. Mohiaddin, D. N. Firmin, and M. H. Yacoub. Asymmetric redirection of flow through the heart. Nature 404:759, 2000. https://d oi.org/10.1038/35008075.

${ }^{21}$ Kim, W. Y., P. G. Walker, E. M. Pedersen, J. K. Poulsen, S. Oyre, K. Houlind, and A. P. Yoganathan. Left ventricular blood flow patterns in normal subjects: a quantitative analysis by three-dimensional magnetic resonance velocity mapping. J. Am. Coll. Cardiol. 26:224-238, 1995. https://d oi.org/10.1016/0735-1097(95)00141-1.

${ }^{22}$ Lancellotti, P., C. Tribouilloy, A. Hagendorff, B. A. Popescu, T. Edvardsen, L. A. Pierard, L. Badano, and J. L. Zamorano. On behalf of the Scientific Document Committee of the European Association of Cardiovascular Imaging: Thor Edvardsen, Oliver Bruder, Bernard Cosyns, 
Erwan Donal, Raluca Dulgheru, Maurizio Galderisi, Patrizio Lancellotti, Denisa Muraru, Koen Nieman, Rosa S, recommendations for the echocardiographic assessment of native valvular regurgitation: an executive summary from the European Association of Cardiovascular Imaging. Eur. Heart J. Cardiovasc. Imaging 14:611-644, 2013. https://doi. org/10.1093/ehjci/jet105.

${ }^{23}$ Lin, B. A., A. S. Forouhar, N. M. Pahlevan, C. A. Anastassiou, P. A. Grayburn, J. D. Thomas, and M. Gharib. Color Doppler jet area overestimates regurgitant volume when multiple jets are present. J. Am. Soc. Echocardiogr. 23:993-1000, 2010. https://doi.org/10.1016/j.echo.2010.06. 011.

${ }^{24}$ Mandinov, L., F. R. Eberli, C. Seiler, and O. M. Hess. Diastolic heart failure. Cardiovasc. Res. 45:813-825, 2000. https://doi.org/10.1016/s0008-6363(99)00399-5.

${ }^{25}$ Matsumura, Y., S. Fukuda, H. Tran, N. L. Greenberg, D. A. Agler, N. Wada, M. Toyono, J. D. Thomas, and T. Shiota. Geometry of the proximal isovelocity surface area in mitral regurgitation by 3-dimensional color Doppler echocardiography: Difference between functional mitral regurgitation and prolapse regurgitation. Am. Heart J. 155:231-238, 2008. https://doi.org/10.1016/j.ahj.2007.09.0 02.

${ }^{26}$ Mohiaddin, R. H. Flow patterns in the dilated ischemic left ventricle studied by MR imaging with velocity vector mapping. J. Magn. Reson. Imaging 5:493-498, 1995. http s://doi.org/10.1002/jmri.1880050503.

${ }^{27}$ Monceau, V., E. Camors, and D. Charlemagne. Physiopathologie du remodelage ventriculaire. MT Cardiol. 2:273-279, 2006.

${ }^{28}$ Muñoz, D. R., J. L. M. Mur, C. Fernández-Golfín, D. C. B. Filho, A. G. Gómez, S. F. Santos, C. L. Rivera, L. M. R. Díaz, E. C. Rojo, and J. L. Z. Gómez. Left ventricular vortices as observed by vector flow mapping: main determinants and their relation to left ventricular filling. Echocardiography 32:96-105, 2015. https://doi.org/10.1111 /echo.12584.

${ }^{29}$ Nishimura, R. A., C. M. Otto, R. O. Bonow, B. A. Carabello, J. P. Erwin, L. A. Fleisher, H. Jneid, M. J. Mack, C. J. McLeod, P. T. O'Gara, V. H. Rigolin, T. M. Sundt, and A. Thompson. 2017 AHA/ACC focused update of the 2014 AHA/ACC guideline for the management of patients with valvular heart disease: a report of the American College of Cardiology/American Heart Association Task Force on Clinical Practice Guidelines. Circulation 2017. https://doi. org/10.1161/CIR.0000000000000503.

${ }^{30}$ Nkomo, V. T., J. M. Gardin, T. N. Skelton, J. S. Gottdiener, C. G. Scott, and M. Enriquez-Sarano. Burden of valvular heart diseases: a population-based study. Lancet 368:1005-1011, 2006. https://doi.org/10.1016/S0140-6736( 06)69208-8.

${ }^{31}$ Okafor, I., V. Raghav, J. F. Condado, P. A. Midha, G. Kumar, and A. P. Yoganathan. Aortic regurgitation generates a kinematic obstruction which hinders left ventricular filling. Ann. Biomed. Eng. 45:1305-1314, 2017. https://d oi.org/10.1007/s10439-017-1790-z.

${ }^{32}$ Papolla, C., J. Adda, A. Rique, G. Habib, and R. Rieu. In vitro quantification of mitral regurgitation of complex geometry by the modified proximal isovelocity surface area method. J. Am. Soc. Echocardiogr. 2020. https://doi.org/10. 1016/j.echo.2020.03.008.

${ }^{33}$ Pedrizzetti, G., and F. Domenichini. Nature optimizes the swirling flow in the human left ventricle. Phys. Rev. Lett. 95:108101, 2005. https://doi.org/10.1103/PhysRevLett.95.1 08101.

${ }^{34}$ Pedrizzetti, G., F. Domenichini, and G. Tonti. On the left ventricular vortex reversal after mitral valve replacement. Ann. Biomed. Eng. 38:769-773, 2010. https://doi.org/10.10 07/s10439-010-9928-2.

${ }^{35}$ Raffel, M., C. E. Willert, S. Wereley, and J. Kompenhans. Particle Image Velocimetry: A Practical Guide (2nd ed.). Berlin: Springer, 2007. https://doi.org/10.1007/978-3-540-7 2308-0.

${ }^{36}$ Saaid, H., J. Voorneveld, C. Schinkel, J. Westenberg, F. Gijsen, P. Segers, P. Verdonck, N. de Jong, J. G. Bosch, S. Kenjeres, and T. Claessens. Tomographic PIV in a model of the left ventricle: 3D flow past biological and mechanical heart valves. J. Biomech. 90:40-49, 2019. https://doi.org/10. 1016/j.jbiomech.2019.04.024.

${ }^{37}$ Stugaard, M., H. Koriyama, K. Katsuki, K. Masuda, T. Asanuma, Y. Takeda, Y. Sakata, K. Itatani, and S. Nakatani. Energy loss in the left ventricle obtained by vector flow mapping as a new quantitative measure of severity of aortic regurgitation: a combined experimental and clinical study. Eur. Heart J. Cardiovasc. Imaging 16:723-730, 2015. https://doi.org/10.1093/ehjci/jev035.

${ }^{38}$ Thomas, L., E. Foster, and N. B. Schiller. Peak mitral inflow velocity predicts mitral regurgitation severity. J. Am. Coll. Cardiol. 31:174-179, 1998. https://doi.org/10.1016/S 0735-1097(97)00454-3.

${ }^{39}$ Vasan Ramachandran, S., and D. Levy. Defining diastolic heart failure. Circulation 101:2118-2121, 2000. https://doi. org/10.1161/01.CIR.101.17.2118.

${ }^{40}$ Yuko, Soyama, Kagiyama Nobuyuki, Vader Justin, Sugahara Masataka, and Gorcsan John. Abstract 14376: The impact of mitral regurgitation on left ventricular energy loss by vector flow mapping in patients with heart failure. Circulation 138:A14376-A14376, 2018. https://doi. org/10.1161/circ.138.suppl_1.14376.

${ }^{41}$ Zoghbi, W. A., D. Adams, R. O. Bonow, M. EnriquezSarano, E. Foster, P. A. Grayburn, R. T. Hahn, Y. Han, J. Hung, R. M. Lang, S. H. Little, D. J. Shah, S. Shernan, P. Thavendiranathan, J. D. Thomas, and N. J. Weissman. Recommendations for noninvasive evaluation of native valvular regurgitation. J. Am. Soc. Echocardiogr. 30:303371, 2017. https://doi.org/10.1016/j.echo.2017.01.007.

${ }^{42}$ Zürcher, F., N. Brugger, S. E. Jahren, S. F. de Marchi, and C. Seiler. Quantification of multiple mitral regurgitant jets: an in vitro validation study comparing two- and three-dimensional proximal isovelocity surface area methods. $J$. Am. Soc. Echocardiogr. 30:511-521, 2017. https://doi.org/ 10.1016/j.echo.2016.12.012.

Publisher's Note Springer Nature remains neutral with regard to jurisdictional claims in published maps and institutional affiliations. 